Acta vet. scand. $1973,14,11-21$.

From the Institute of Pharmacology, University of Oslo, Blindern, Norway.

\title{
MICROSOMAL DRUG OXIDATION
}

FUNCTION, INDUCTION, AND INHIBITION OF THE CYTOCHROME P450-SYSTEM

By

Erik Dybing

Most drugs undergo metabolic changes in the organism, i.e. chemical alterations to substances different from the parent drug. From the middle of the nineteen-fifties it is known that these changes are catalyzed by enzymes and that the reactions usually take place in the microsomal fraction of the liver. A wide variety of drugs are enzymatically oxidized by this microsomal system in the presence of molecular oxygen and NADPH. The need for both oxygen and NADPH should indicate that NADPH reduces a factor in the liver microsomes which together with oxygen forms an active oxygen-complex and that this complex transfers oxygen to the drug molecule. The factor that participates in such reactions is now believed to be a particular hemoprotein called cytochrome $\mathrm{P} 450$; in its reduced form this cytochrome binds carbon monoxide to give an extinction maximum at $450 \mathrm{~nm}$. Cytochrome $\mathbf{P} 450$ plays an important rôle in the oxidative metabolism of drugs, where it functions as terminal oxygenase.

Knowledge of the drug metabolizing systems and of the possibilities of alteration of the activity of these systems are of great importance for the evaluation of drug therapy and drug toxicity. In the following, the functions of the cytochrome P450-system will be outlined. In addition, some biochemical aspects of the induction and inhibition of the cytochrome P450-system will be mentioned. 
The functions of the cytochrome P450-system

The cytochrome P450-system is remarkable in its ability to oxidize such a wide variety of endogenous and exogenous substances as cholesterol, steroids, fatty acids, drugs, polycyclic hydrocarbons, and xenobiotics. Because of the need for oxygen this enzyme system has been termed mixed function oxidase or mono-oxygenase. It is still not known whether these reactions are catalyzed by one single enzyme or by a family of different enzyme systems.

In most oxidation reactions that occur in the liver microsomes the drug substrate is first bound to the oxidized form of cytochrome P450 (Fig. 1, upper right). This substrate-P450complex is then reduced by an electron from a flavoprotein called NADPH cytochrome c reductase whereby a reduced substrate-P450-complex is formed. This complex in turn reacts with oxygen so that a substrate- $\mathrm{P} 450-\mathrm{O}_{2}$-complex is formed. The next step is not yet quite clear, but it is believed that a second electron reduces the substrate-P450- $\mathrm{O}_{2}$-complex to an active oxygen-intermediate, which then decomposes forming the oxidized substrate

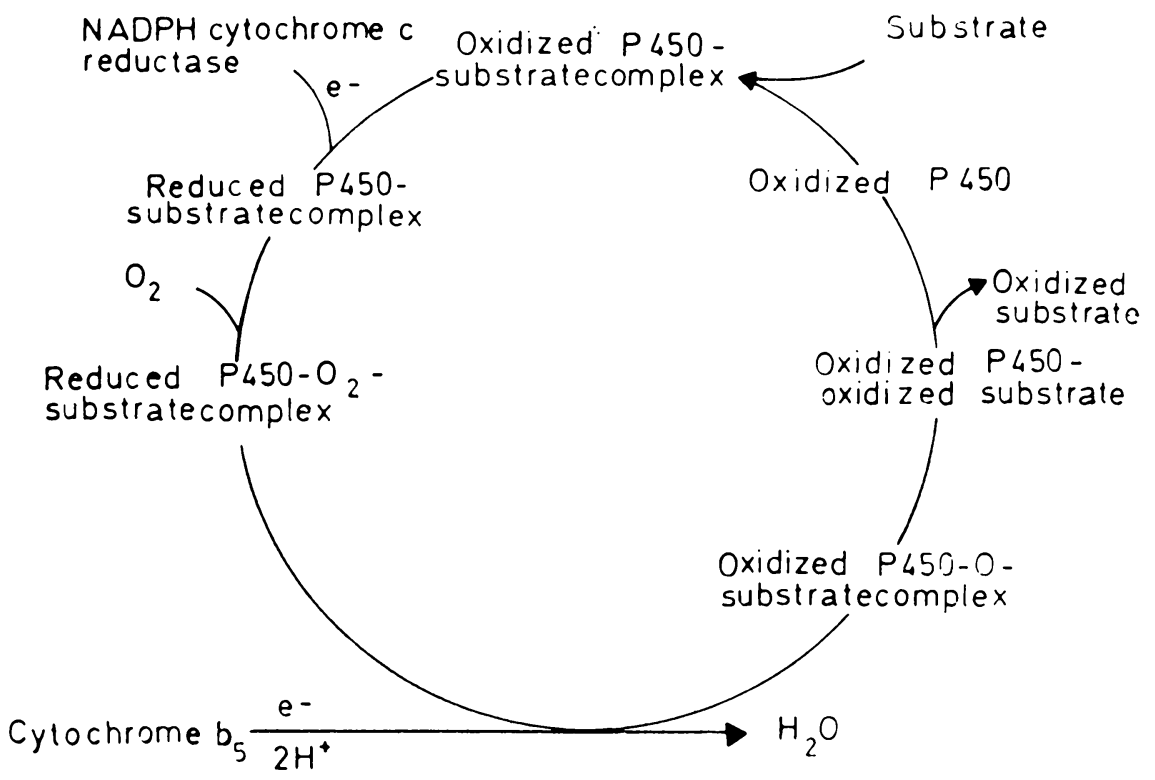

Fig u r e 1. ESTABROOK's schema for the transport of electrons in the cytochrome P450-mediated oxidation of drugs (from Gillette et al., Ann. Rev. Pharmacol. 1972, 12, 57). 


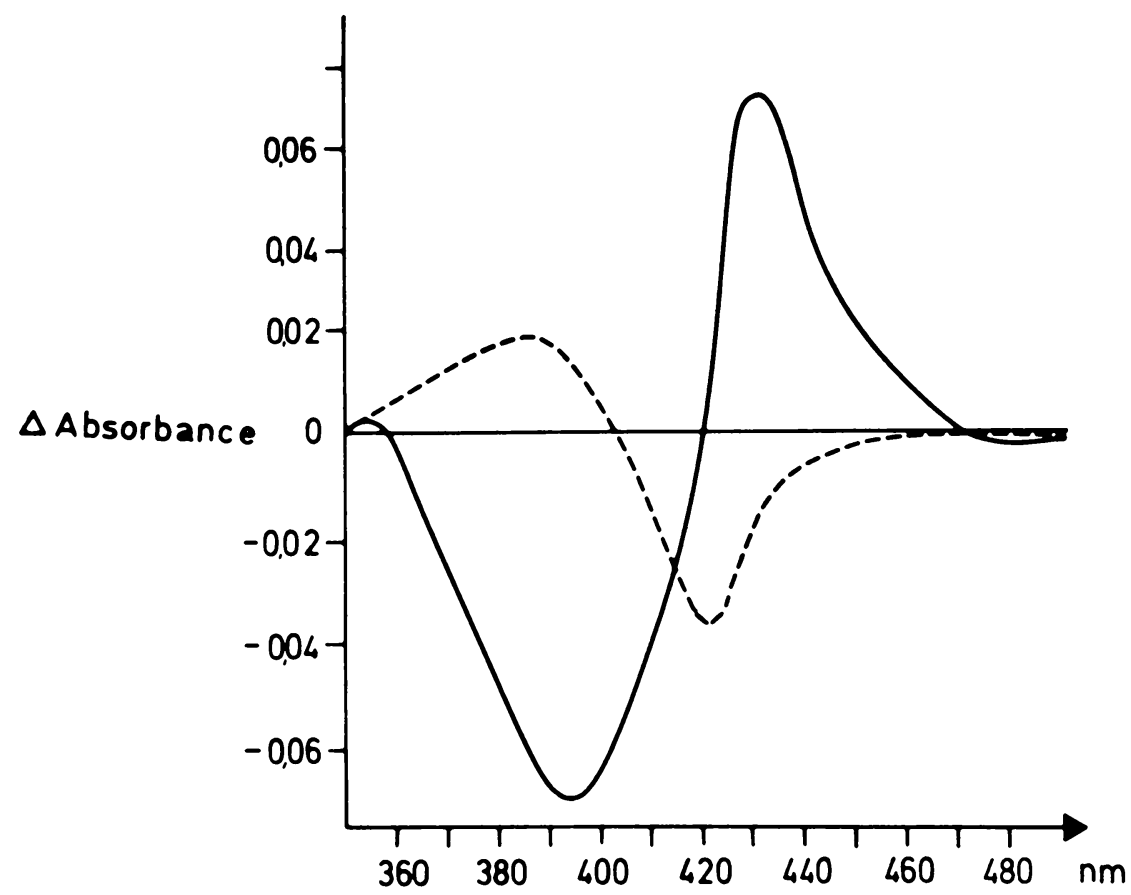

Figure 2. Type I (hexobarbital, -....-) and type II (aniline, ) difference spectra after addition of substrates to microsomal suspensions in the presence of oxygen (from Sasame \& Gillette, Mol.

Pharmacol. 1969, 5, 123).

at the same time as the cytochrome $\mathrm{P450}$ is reoxidized. The second electron needed for this reaction to take place is believed to be furnished from another microsomal cytochrome called cytochrome $b_{5}$. Rate-limiting for the oxidation of a great number of drugs is the NADPH reduction of the cytochrome P450-substratecomplex.

If substrates are added to suspensions of microsomes in the presence of oxygen but without NADPH, difference spectra can be recorded as a sign of the substrate-induced change of the absolute spectrum of the suspension. Depending on the substrate used, these spectral binding characteristics can be regarded as belonging to one of two types (Fig. 2): Type I (represented by hexobarbital) and type II (represented by aniline). It is believed that these two types of spectral changes are caused by the binding of the substrates to one of two sites on cytochrome P450. Most substrates bind to site I, whereas site II is the binding site for 
a few substrates and $\mathrm{O}_{2}$ (as well as $\mathrm{CO}$ ). Oxygen must be bound to site II for other substrates to be bound to site I (allosteric effect). The type I spectral change is not elicited by the substrates binding to the heme portion of the hemoprotein, but by modification of the already existing ligand between the heme and the protein. The type II spectral change has been shown to be a ferrihemochrome, i.e. evoked by the binding of the substrate to the heme iron. A third type of spectral change called reversed type I (or modified type II) has also been demonstrated. This type of spectral change has an absorption maximum at $420 \mathrm{~nm}$ and minimum at $392 \mathrm{~nm}$, and is believed to develop from interaction of lipid-soluble substances with the substrate-bound form of cytochrome P450 at a binding site different from site I. The degree of spectral changes is dependent on the amount of drugcytochrome complex present and usually follows enzyme kinetic laws.

The concentration and activity of cytochrome P450 may vary greatly between the different species, between individuals, and also in the same individual depending on age, chemical or hormonal influences etc. The rate of oxidation is more dependent on the activity than the amount of the cytochrome (the same amount of cytochrome does not always lead to the same rate of metabolism). Known sex and species variations are perhaps caused by changes in activity; at any rate the differences in the rate of metabolism noted within the same species can be explained by differences in activities. The cytochrome P450 found in human liver does not show any difference in spectral characteristics compared to the cytochrome P450 from livers of laboratory animals. It is not possible to apply the same enzyme kinetic rules on the microsomal oxidation system as those used on soluble enzyme systems. Changes in the composition (especially the lipid component) and the three-dimensional structure of the endoplasmic reticulum may lead to changes in the activity of this electron-transport system.

\section{Induction of the cytochrome P450-system}

More than two hundred different chemical substances can after pretreatment of laboratory animals increase the activity of their microsomal oxidation system, and are thereby able to change the degree and duration of drug action. These substances 
may be very different in respect to chemical structure and pharmacological action.

Pretreatment of animals with phenobarbital will increase the rate of metabolism of a great number of drugs, at the same time the activity of NADPH cytochrome c reductase and the concentration of cytochrome $\mathrm{P} 450$ in the liver are increased. The degree of the type I and type II spectral changes also increases, and the synthesis of heme and phospholipids in the liver is enhanced. This effect of phenobarbital is not indirectly mediated through the endocrine system, induction of microsomal enzymes has been shown both in isolated, perfused livers and in cell cultures from foetal liver.

Substances such as 3-methylcholanthrene and 3,4-benzpyrene are also capable of increasing the metabolism of drugs by the miorosomal oxidation system, but there are differences in the induction pattern compared to that seen after phenobarbital. 3-methylcholanthrene acts more selectively, i.e. increases the metabolism of a fewer number of drugs than phenobarbital. 3-methylcholanthrene increases the amount of cytochrome P450, whereas the amount of NADPH cytochrome c reductase does not change. The interval between application and maximal induction amounts to hours for the polycyclic hydrocarbons, whereas it takes days after phenobarbital (Fig. 3).
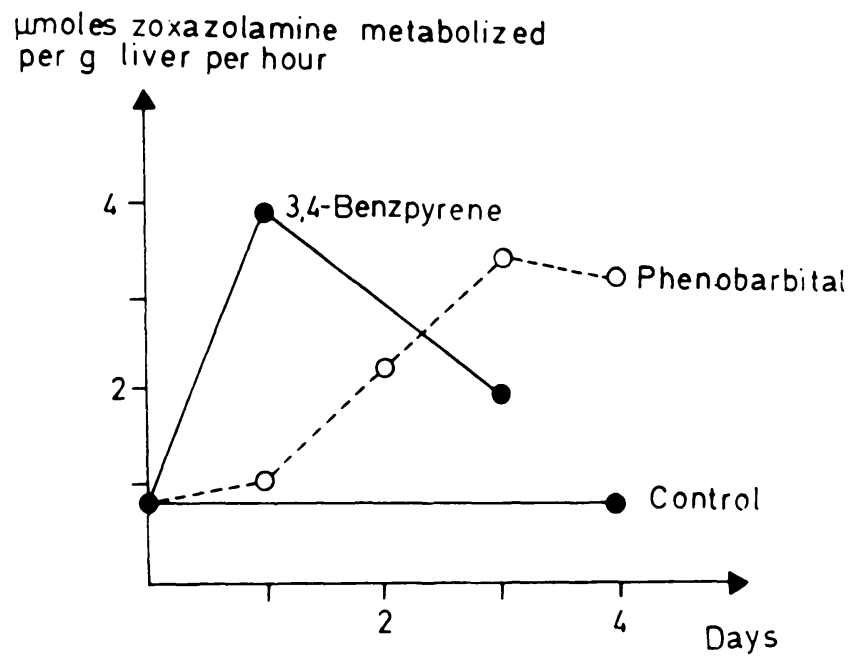

Figure 3. Difference in time in the induction of the metabolism of zoxazolamine after 3,4-benzpyrene and phenobarbital (from Conney \& Burns, Adv. Pharmacol. 1962, 1, 31). 
When 3-methylcholanthrene and phenobarbital are administered at the same time, additive effects are seen on the metabolism of substances which separately are induced by one of the two drugs. The complex of $\mathrm{CO}$ and reduced cytochrome P450 from normal or phenobarbital-pretreated animals has a sharp absorption peak at $450 \mathrm{~nm}$, whereas the microsomes from 3-methylcholanthrene-pretreated animals treated with CO develop a maximum at 446 to $448 \mathrm{~nm}$. This spectral change is not seen after direct addition of 3-methylcholanthrene in vitro, but seems to be a result of new synthesis of a hemoprotein different from cytochrome $\mathrm{P} 450$ (called $\mathrm{P} 448$ ). It should be pointed out that there does not seem to be any connection between the inductory and carcinogenic effects of the polycyclic hydrocarbons.

Foetal and newborn animals have very low amounts of microsomal drug oxidizing enzymes. The basal level of these enzymes changes very little in the prenatal period, whereas the ability to respond to inducers changes dramatioally. Early in the foetal period no reaction after phenobarbital application can be seen, but in the latest part of gestation (4-5 days before birth in rabbits) there is induction of the enzymes after phenobarbital. There is evidently a period in the later part of gestation where the basal level of enzyme activity does not change, but where the response to inducers develop. This points to the fact that the maintenance of basal enzyme activity and induction are under separate control. Different theoretical models for control at the level of translation and transcription have been proposed. A supposedly important criterion for induction, at any rate for translation, is that the inducing substance should be a substrate for the microsomal oxidizing system. Old animals also have lower microsomal enzyme activity than younger, adult animals; also, they do not respond as well as younger animals to phenobarbital.

Pretreatment of animals with phenobarbital for 3-6 days leads to an increase in liver weight (about $30 \%$ ), in the liver weight/body weight ratio, in the amount of microsomal protein (about $40 \%$ ), and in the rate of synthesis of microsomal protein. Inducing substances will also increase the degree of regeneration after partial hepatectomy. The increase in liver weight in connection with induction is brought about both by hepatocellular hyperplasia $(15 \%)$ and hypertrophy $(20 \%)$. Phenobarbital and similar compounds are seen to cause a massive proliferation of 
the endoplasmic reticulum, whereas after 3-methylcholanthrene no such change can be demonstrated. However, there is no correlation with time in the proliferation of the endoplasmic reticulum and the augmentation of the microsomal enzyme activities after phenobarbital.

Rats treated with 3-methylcholanthrene increase their amount of nuclear RNA approx. $40 \%$, and the nuclear RNA is more active in directing protein synthesis than RNA from control animals. Further an increase can be seen of 50-100\% in the in vitro-incorporation of orotic acid and cytidine phosphate into RNA from the 3-methylcholanthrene treated animals. Animals pretreated with phenobarbital of 3-methylcholanthrene incorporate radioactive amino acids into miorosomal protein at a greater velocity than do control animals; this effect can be blocked with actinomycin D. From this it was believed that the increase in amount of microsomal protein and enzyme activities could be explained from increased synthesis only. However, turnover studies have revealed that especially phenobarbital has a profound effect also on the breakdown of microsomal protein. Repeated doses of phenobarbital thus leads to an increase of microsomal protein through a dual mechanism, through increased protein synthesis and decreased protein breakdown. At the same time as inducers increase the amount of microsomal protein they also increase the amount of phospholipids. This is mostly a result of great reduction in the catabolism of phospholipids.

3-methylcholanthrene also stimulates microsomal oxidizing enzymes in the kidney, small intestine, and lung in addition to the liver. Recently, drug oxidizing enzymes (AHH, arylhydrocarbon hydroxylase) have been demonstrated in leucocytes, and these enzymes can also be induced. In the normal human placenta there is low, but demonstrable activity of drug metabolizing enzymes. In the placenta from cigarette smokers this activity is several times higher than in the placenta from non-smokers; benzpyrene has been claimed to be the causative inducing agent.

The influence of chemicals on the drug metabolizing enzyme systems has wide implications in pharmacology and toxicology. Prolonged treatment with phenobarbital may necessitate an increase in the dose of other drugs in order to achieve a therapeutic effect of these. If phenobarbital is discontinued the induction will terminate and the high drug doses can lead to symptoms of toxicity. Continuous treatment with a drug can also 


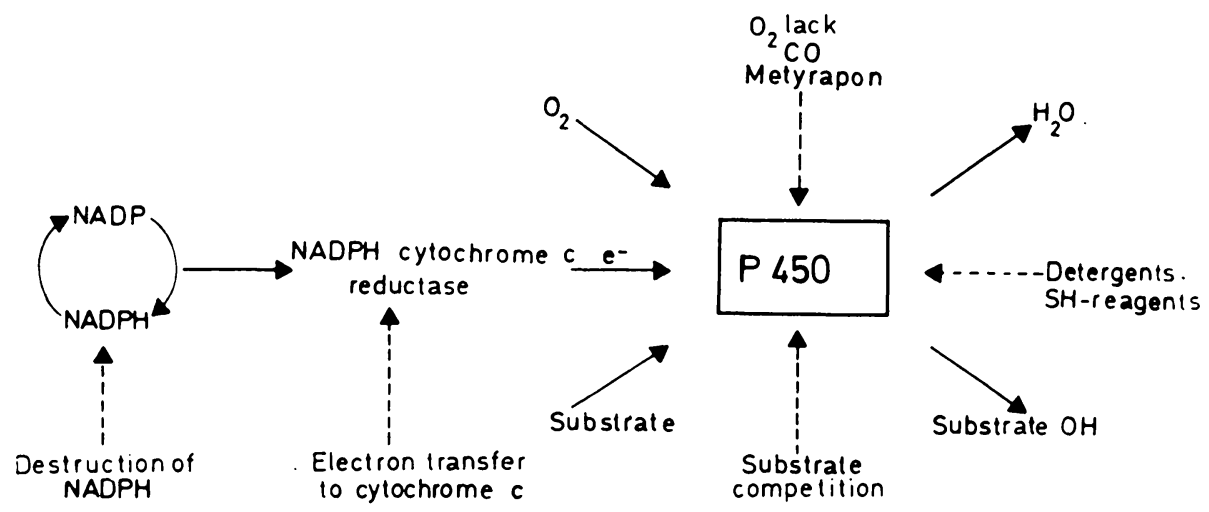

Figure 4. Schematic presentation of microsomal drug oxidation and the action of inhibitors of the cytochrome P450-system (from Netter, Arzneimittel-Forsch. 1972, 22, 285).

induce microsomal enzymes which catalyze its own breakdown, the so-called auto-induction. This mechanism may be an explanation to the apparent tolerance to drugs (pseudo-tolerance). The auto-induction phenomenon must also be considered when judging results from chronic toxicity experiments.

Inhibition of the cytochrome P/50-system

A great number of times the use of enzyme inhibitors has proved fruitful when biological reaction mechanisms are being investigated. The inhibition of drug metabolism both in vivo and in vitro has also helped to elucidate the principles of microsomal oxidation.

From Fig. 4 a schematic representation of the point of attack of inhibitors of drug oxidation can be seen. Theoretically a destruction of NADPH (enzymatic breakdown via NADase, pyrophosphatase) can lead to inhibition of microsomal oxidation. The electron transfer to cytochrome P4j0 proceeds at a slower rate than to cytochrome $c$ (the flavoprotein was named NADPH cytochrome c reductase before its function in drug oxidation was known). In vitro addition of cytochrome $c$ to washed microsomes leads to diversion of electrons to cytochrome c, the reduction of cytochrome P450 is not brought about, and the drug oxidation is inhibited. 
Direct inhibition of cytochrome $\mathrm{P} 450$ can be seen from the right part of Fig. 4. Substrates that are metabolized by the cytochrome P450-system can mutually inhibit the metabolism of each other through competition for binding to cytochrome P450. One of the first substances to be used that showed inhibition of drug metabolism both in vivo and in vitro was SKF 525-A (diethylaminethyl diphenylvalerate). It was shown that SKF 525-A is a substrate for the cytochrome P450-system. If SKF 525-A is added together with another substrate for the microsomal oxidizing system to incubation medium before addition of microsomes, SKF 525-A inhibits competitively the metabolism of the other substrate. Similar kinetic data have been found with a great number of drugs that are metabolized by the cytochrome P450-system. Later it has been found that also SKF 525-A can show non-competitive inhibition of drug microsomal oxidation. This is seen if miorosomes are preincubated with SKF 525-A and NADPH, and is ascribed to the formation of a stable cytochrome P450- $\mathrm{O}_{2}$-complex after metabolic conversion of SKF 525-A. The in vivo situation is even more complex; it has been shown that SKF 525-A can reduce cellular permeability, give diminished blood circulation in the liver, and perhaps lead to decreased absorption of drugs in the intestine.

Carbon monoxide is more strongly bound to iron in the hemoprotein than is oxygen, it will therefore impede substrate binding and thus give inhibition of drug oxidation. Monochromatic light of wavelength $450 \mathrm{~nm}$ will reverse this CO-induced inhibition. Lack of oxygen acts in the same manner as CO. Metyrapon (Metapiron $\AA$ ), an inhibitor of steroid hydroxylases in the adrenal cortex, also inhibits microsomal drug oxidation, much in the same manner as CO. Metyrapon leads to decreased oxidation of several drugs both in vivo and in the isolated, perfused liver as well as it gives competitive inhibition in vitro. Metyrapon (as $\mathrm{CO}$ ) binds to site II of cytochrome P450 and thereby prevents substrate binding to site $I$.

Unspecific inhibition of drug oxidation is seen after addition of detergents, presumably by destruction of the lipid membrane. Attempts to solubilize the microsomal enzymes have proven very difficult, the use of surface-active substances has mostly resulted in total loss of enzymatic activity. Sulfhydryl-groups are evidently necessary for drug metabolism. Blocking of SH-groups with p-chloromercuribenzoate (PCMB) leads to reversible in- 
hibition of for instance microsomal demethylation; this effect can be abolished by the addition of $1(+)$-cysteine. The function of $\mathrm{SH}$-groups in the oxidation process remains to be explained.

\section{Conclusion}

The discovery of the cytochrome P450-system and the elucidation of its functions are not only of theoretical interest. The above mentioned findings are also important for drug therapy. Clinical-pharmacological investigations have proven that the principles for the metabolism of drugs also apply in the clinical situation. The microsomal drug oxidizing system is functionally similar in humans and animals; human liver contains cytochromes $\mathrm{P} 450$ and $\mathrm{b}_{5}$, although at a lower concentration than in animals. Induction and inhibition of the cytochrome P450-system leading to altered drug metabolism may modify the clinical effect of drugs. Manipulation with the activity of this system gives one the possibility to bring about enhanced or decreased metabolism of drugs or endogenous substances.

\section{LITERATURE}

1. Anders, M. W.: Enhancement and inhibition of drug metabolism. Ann. Rev. Pharmacol. 1971, 11, 37-56.

2. Conney, A. H.: Pharmacological implications of microsomal enzyme induction. Pharmacol. Rev. 1967, 19, 317-366.

3. Gillette, J. R., A. H. Conney, G. J. Cosmides, R. W. Estabrook, J. R. Fouts \& G. J. Mannering: Microsomes and Drug Oxidations. Acad. Press, New York and London 1969.

4. Gillette, J. R., D. C. Davis \& H. A. Sasame: CytochromeP-450 and its role in drug metabolism. Ann. Rev. Pharmacol. 1972, 12, 57-84.

5. Gram, T. E. \& J. R. Gillette: Biotransformation of drugs. In Fundamentals of Biochemical Pharmacology. Pergamon Press, Oxford, New York and Toronto 1971, pp. 571-609.

6. Kuntzman, R.: Drugs and enzyme induction. Ann. Rev. Pharmacol. $1969,9,21-36$.

7. Mannering, G. J.: Inhibition of drug metabolism. In Handbook in Experimental Pharmacology, vol. XXVIII. Concepts in Biochemical Pharmacology, part 2. Springer Verlag, New York 1971, pp. 452-476.

8. Netter, $K$. J.: Grundlagen des Arzneimittelstoffwechsels. Arzneimittel-Forsch. 1972, 22, 285-292.

9. Orrenius, S.: Molecular aspects of drug metabolism. Acta pharmacol. (Kbh.) 1971, 29, Suppl. 3, 191-202. 
10. Rugstad, $H$. E.: Induksjon og hemming av legemiddelmetaboliserende enzymer. Betydning for farmakoterapi, toksisitet og klinisk utprøvning. T. norske Lægeforen. 1969, 89, 172176.

11. Uehleke, H.: Pathophysiologische und biochemische Grundlagen des Arzneimittelstoff wechsels. In Klinische Pharmakologie und Pharmakotherapie. Urban-Schwarzenberg-Verlag, München und Berlin 1971, pp. 115-131.

12. Williams, R. T.: Pathways of drug metabolism. In Handbook of Experimental Pharmacology, vol. XXVIII. Concepts in Biochemical Pharmacology, part 2. Springer Verlag, New York 1971, pp. 226--241.

Reprints may be requested from: Institute of Pharmacology, Veterinary College of Norway, Postbox 8146, Oslo Dep., Oslo 1, Norway. 